\title{
SARS-CoV-2 infection dynamics in lungs of African green monkeys
}

2 Emily Speranza ${ }^{1,4}$, Brandi N. Williamson ${ }^{1}$, Friederike Feldmann², Gail L. Sturdevant ${ }^{1}$, Lizzette

3 Pérez- Pérez ${ }^{1}$, Kimberly Mead-White ${ }^{1}$, Brian J. Smith², Jamie Lovaglio ${ }^{2}$, Craig Martens ${ }^{3}$, Vincent

4 J. Munster ${ }^{1}$, Atsushi Okumura ${ }^{1}$, Carl Shaia ${ }^{2}$, Heinz Feldmann ${ }^{1}$, Sonja M. Best ${ }^{1}$, Emmie de Wit $^{1^{*}}$

5

${ }^{1}$ Laboratory of Virology, ${ }^{2}$ Rocky Mountain Veterinary Branch and ${ }^{3}$ Research Technologies

Branch, Division of Intramural Research, National Institute of Allergy and Infectious Diseases, National Institutes of Health, Hamilton, MT, United States of America, ${ }^{4}$ Laboratory of Immune System Biology, Lymphocyte Biology Section, Division of Intramural Research, National Institute of Allergy and Infectious Diseases, National Institutes of Health, Bethesda, MD, United States of America

*Corresponding author: emmie.dewit@nih.gov

Keywords: African green monkey, SARS-CoV-2, single cell sequencing, virus replication, respiratory tract, animal model, macrophages, pneumocytes

\section{Summary}

Detailed knowledge about the dynamics of SARS-CoV-2 infection is important for unraveling the viral and host factors that contribute to COVID-19 pathogenesis. Old-World nonhuman primates recapitulate mild-moderate COVID-19 cases, thereby serving as important pathogenesis models. We compared African green monkeys inoculated with SARS-CoV-2 or inactivated virus to study the dynamics of virus replication throughout the respiratory tract. RNA sequencing of single cells from the lungs and mediastinal lymph nodes allowed a high-resolution analysis of 
1 virus replication and host responses over time. Viral replication was mainly localized to the

2 lower respiratory tract, with evidence of replication in the pneumocytes. Macrophages were

3 found to play a role in initiating a pro-inflammatory state in the lungs, while also interacting with

4 infected pneumocytes. Our dataset provides a detailed view of changes in host and virus

5 replication dynamics over the course of mild COVID-19 and serves as a valuable resource to

6 identify therapeutic targets.

7

8

\section{Introduction}

A wealth of clinical and laboratory studies have been reported concerning severe acute respiratory syndrome coronavirus 2 (SARS-CoV-2), the causative agent of coronavirus disease 2019 (COVID-19) (Ge et al., 2020; Tay et al., 2020). Among the many unanswered questions that remain, key issues involve the dynamics of SARS-CoV-2 infection, including the identity of the cells that support active virus replication and the immune response to infection. Though multiple cell types in the respiratory tract have been reported to express the critical receptor (ACE2) needed for entry, as well as the protease (TMRPSS2) needed to initiate replication (Qi et al., 2020; Sungnak et al., 2020), it is not clear which of these cell types the virus actively replicates in. One study, using a new algorithm (Viral Track) to detect viral reads in single cell sequencing samples, suggested that both epithelial cells and macrophages contain viral RNA in human bronchoalveolar lavage fluid (BALF) (Bost et al., 2020). However, since the presence of genomic RNA (gRNA) alone does not equate to productive virus infection, this study did not address whether this detection of RNA was the result of virus replication in these cells.

Beyond viral replication dynamics, a thorough study of the host response to infection at the major site of virus replication, the lungs, is needed to better understand potential causes of organ damage and targets for therapeutic intervention. The use of single cell technologies such as multi-parameter flow cytometry and single cell sequencing allow analysis of individual cell 
1 states. Sequencing of single cells in BALF (Liao et al., 2020) and upper respiratory tract swabs

2 (Chua et al., 2020) collected from COVID-19 patients detected markers of severe disease in

3 hospitalized individuals. Others have examined the immune response to infection by utilizing

4 single cell sequencing to profile PBMC samples from COVID-19 patients (Wen et al., 2020; Wilk

5 et al., 2020). However, both approaches have limited power to address the dynamics of

6 infection, the host response in the lungs, the main site of virus replication and disease

7 pathogenesis, and neither provides insight into the immune response in the draining lymph

8 nodes.

9 The limitations of human studies with respect to time of sampling relative to exposure, differences in exposure dose and route, and capacity for deeply analyzing tissues can be overcome using animal models, albeit currently at the cost of not fully replicating the severe disease observed in humans. Indeed, multiple animal models of SARS-CoV-2 infection are being developed to test therapeutics and vaccines and well as to better understand the dynamics of COVID-19 disease progression and the immune response to infection in a timeresolved manner. Non-human primates are commonly used as models for infection and pathogenesis since they often recapitulate human disease. African green monkeys (AGM) are

17 a commonly used non-human primate model for studies of respiratory viruses, including SARSCoV (McAuliffe et al., 2004). Recently, two studies showed that inoculation of AGM with SARSCoV-2 results in mild respiratory disease with virus detected in the upper and lower respiratory tract, suggesting this is a suitable nonhuman primate disease model to study viral infection and host response dynamics (Hartman et al., 2020; Woolsey et al., 2020).

To address some of the major questions about SARS-CoV-2 replication and host

23 response, we used traditional virological methods, single cell RNA sequencing, and

24 immunohistopathology. Our combined approach, using inoculation with infectious as well as

25 inactivated SARS-CoV-2 helped determine which cells the virus is replicating in and assess the 26 host response to virus replication. Together, the result is an emerging picture of the viral and 
1 immune events associated with mild COVID-19 disease, aiding the understanding some of the

2 dynamics of SARS-CoV-2 infection with very high resolution.

3

4

\section{Results}

sgRNA detection reveals active virus replication in the respiratory tract of SARS-CoV-2 infected AGM

To study SARS-CoV-2 infection and its consequences, two groups of four African green monkeys were inoculated with replication-competent virus, while two control animals were inoculated with virus inactivated by gamma-irradiation. Clinical signs in the control animals were limited to reduced appetite likely as a response to repeated anesthesia (Table 1). Tachypnea was observed in five of eight animals inoculated with infectious SARS-CoV-2. In these animals, disease was mild to moderate and transient, with animals recovering between 5 and 9 days post-inoculation (dpi) (Fig. S1A and Table 1). At 1, 3, 5, 7, and 10 dpi we collected nose, throat and rectal swabs from all animals. Levels of viral genomic RNA (gRNA) in nose and throat swabs were high after inoculation with SARS-CoV-2 and declined over time. Rectal swabs were positive at most time points in one animal with a severely reduced appetite (AGM8) (Fig. 1).

Surprisingly, nasal, although not throat or rectal, swabs from control animals inoculated with gamma-irradiated virus contained high amounts of gRNA at $1 \mathrm{dpi}$ and were still positive at 3 dpi. To determine whether detection of subgenomic RNA (sgRNA) would be able to distinguish between RNA originating from the inoculum from that derived from replicating virus, all swabs positive for gRNA were run in a qRT-PCR to detect subgenomic RNA (sgRNA). Although sgRNA was present at high levels in both inocula, sgRNA could not be detected in swabs collected from control animals but could in nose and throat swabs from animals inoculated with infectious virus (Fig. 1), indicating that sgRNA likely reflects that virus replication occurred. Infectious virus could mainly be detected by virus titration early after inoculation in nose and throat swabs; no infectious virus could be detected in rectal swabs (Fig. 1). As a measure of 
1 virus replication in the lower respiratory tract, we collected BALF from the two control animals at

21 and 3 dpi and at 1, 3, and 5 dpi from the four SARS-CoV-2 infected animals euthanized at 10

3 dpi. gRNA could be detected on 1 and 3 dpi in one of the two control animals; however, sgRNA

4 could not be detected. High levels of gRNA and sgRNA were detected in BALF from the four

5 infected animals, in line with detection of infectious virus through $5 \mathrm{dpi}$ (Fig. 1).

6

\section{Assessment of SARS-CoV-2 replication in various tissues}

At $3 \mathrm{dpi}$, the two control animals and four of the SARS-CoV-2 infected animals were euthanized. The remaining four SARS-CoV-2 animals were euthanized at $10 \mathrm{dpi}$. Upon necropsy, lungs were examined for gross lesions. No abnormalities were detected in the lungs of the two control animals. At $3 \mathrm{dpi}$, all four animals inoculated with active SARS-CoV-2 showed varying degrees of gross lung lesions and enlarged mediastinal lymph nodes (Table 1 and Fig. S1B). By $10 \mathrm{dpi}$, one animal did not show gross abnormalities; whereas, the other three animals showed gross lung lesions and enlarged mediastinal lymph nodes (Table 1 and Fig.S1B). Tissue samples from these animals were assessed for the presence of gRNA and sgRNA. Viral gRNA loads were highest in samples collected from the lung lobes and were higher at $3 \mathrm{dpi}$ than 10 dpi. Despite high levels of sgRNA in lung tissue through $10 \mathrm{dpi}$, virus could only be isolated at $3 \mathrm{dpi}$ (Fig. S1C and Table S1), indicating that in tissue, sgRNA is a much more sensitive detection method than virus isolation in tissue culture. Analysis of other respiratory tract tissues showed that although gRNA can be detected in all tested sites early after inoculation, sgRNA can be detected consistently only in the trachea and right bronchus (Fig. S1D). Thus, in the respiratory tract of AGM, the lung parenchyma is the main site of virus replication.

We also analyzed tissues of the gastrointestinal (GI) tract for the presence of viral RNA. gRNA alone could be detected in the GI tract of several animals after inoculation with SARSCoV-2 at 3 dpi and 10 dpi. However, in AGM8, the animal with severely reduced appetite, high 
1 levels of both gRNA and sgRNA could be detected in duodenum, jejunum, ileum, cecum and

2 colon (Fig. S1E) and virus was isolated from the ileum and cecum (Table S1). Histologically, the

3 intestinal tract from this animal appeared normal; yet, immunohistochemistry $(\mathrm{IHC})$ revealed

4 epithelial cells containing SARS-CoV-2 antigen in the ileum of AGM8 (Fig. S2A-C).

Histological analysis of the lungs of the two control animals showed no abnormalities

6

(Fig. 2). The lungs of the four animals inoculated with SARS-CoV-2 and euthanized at $3 \mathrm{dpi}$ showed subtle alveolar thickening, indicative of an early inflammatory response (Fig. 2). Viral antigen could be detected by immunohistochemistry in type I pneumocytes and alveolar macrophages of all four animals. The alveolar thickening was still visible in the four animals inoculated with SARS-CoV-2 and euthanized at $10 \mathrm{dpi}$. Two of these animals showed histopathological changes consistent with interstitial pneumonia frequently centered on terminal bronchioles and early lesions in terminal airways resembling obstructive bronchiolitis (Fig. 2). At this time, viral antigen could only be detected in type I pneumocytes and alveolar macrophages of one of four animals (AGM10). Three of four mediastinal lymph nodes from the $10 \mathrm{dpi}$ samples exhibited a mild to moderate follicular hyperplasia and all four animals' lymph nodes exhibited rare mononuclear cell immunoreactivity (Fig S2D-E).

\section{RNA sequencing of single cells from lungs of infected AGM}

On the day of necropsy ( 3 dpi for the two control animals inoculated with gammairradiated virus, 3 and 10 dpi for the eight animals that received SARS-CoV-2), we collected sections of the lungs of each animal that contained an active lesion, except in animals where gross lung lesions were not observed at necropsy (Table 1). These sections were processed directly following necropsy into single cell suspensions and single cell RNA sequencing was conducted using cDNA generated immediately without freezing or fixation of the cells, thereby allowing collection of whole cell data. This allowed for high-quality single cell data to be collected with a high fraction of reads in cells (> 80\%) and a low fraction of cells enriched in 
1 mitochondrial genes (<5\%). Uniform manifold approximation and projection (UMAP) was used

2 to display the data with each cell annotated with its likely cell identity (Fig. 3A). For the latter

3 analysis we developed a correlation-based method that uses transcriptional profiles from

4 annotated lung tissue and determines the most likely identity for an individual cell or a cluster of

5 cells (see methods). To validate the results, we built a marker gene set for each cell type of

6 interest, showing that the gene expression profile matched the cell type annotation (Fig. 3B).

7 Using these assignments, we compared the percentage of cells in each sample belonging to

8 each annotation and found a trend towards an increase in plasma cells as well as an increase in

9 the number of pneumocytes and dividing cells as infection progressed (Fig. S3). This is consistent with the histology results showing an influx of inflammatory cells and type II pneumocyte hyperplasia at $10 \mathrm{dpi}$ (Fig. 2F).

Since SARS-CoV-2 is a poly-adenylated virus, with such modification of both the genome (gRNA) and the transcripts (sgRNA), we could map reads to both the AGM and the SARS-CoV-2 genome and determine which cell types contained viral RNA. The scRNA-Seq data showed a similar pattern to the qRT-PCR data described with the highest percentage of positive cells at $3 \mathrm{dpi}$, and a decrease by $10 \mathrm{dpi}$ (Fig S1C); no viral RNA was detected in the animals inoculated with gamma-irradiated virus (Figure $3 \mathrm{C}$ and $\mathrm{D}$ ). We then parsed these data down to individual cell types. At 3 dpi, viral RNA could be detected in several cell types, with the macrophage population having the highest percentage of cells positive for viral RNA (Figure 3E). The viral RNA in these cells could be due to virus replication, phagocytosis of infected

21 cells, abortive infections, or having virus particles partitioned with cells during GEM creation in

22 the 10X genomics processing steps. To discriminate between cells supporting active virus

23 replication vs. those containing viral RNA due to other processes, we looked at the distribution

24 of reads across the genome. Due to the 3' bias of sequencing with the 10X platform, we saw an

25 expected enrichment of reads in the 3' end (Figure S4A), with a bias to the location of the N

26 gene in the genome. Additionally, in the pseudo-bulk data read pileups, we were able to detect 
1 small enrichments of reads in specific areas at the most 3' end of all the transcripts, including

2 ORF1ab, as well as pileups in the first 5000 base pairs (bp) of the ORF1ab region (Figure S4B).

3 These points of enrichment in the first $5000 \mathrm{bp}$ matched to locations of non-canonical sgRNA

4 formed by a jump of the RNA-dependent RNA polymerase in ORF1ab to N in the SARS-CoV-2

5 transcriptome and are suggestive of actively replicating virus (Kim et al., 2020). Based on these

6 findings, we examined each cell type for reads showing such evidence of subgenomic

7 transcripts involving a region other than N. Only one annotated cell type, pneumocytes, had a

8 similar proportion of cells that were positive for the $\mathrm{N}$ gene and for ORF1ab that was also an

9 abnormally high ratio of ORF1ab to $\mathrm{N}$ compared to all other cell types (Fig. 3F). This suggests

10 that despite many cell types containing viral RNA, the pneumocytes were likely the dominant

11 cell type supporting productive viral replication. To examine this hypothesis further, we

12 performed IHC and in-situ hybridization (ISH) on lung tissues at 3 dpi. Although along with

13 pneumocytes, a few macrophages were positive for viral antigen by $\mathrm{IHC}(\mathrm{Fig} .2 \mathrm{H})$, only

14 pneumocytes were positive by ISH against the viral genome, consistent with the notion that this

15 is the only cell type analyzed supporting active virus replication (Fig. 3G).

\section{Infection-related changes in lung cell transcriptional states}

To gain insight into the biological effects of SARS-CoV-2 infection on diverse cells in the lungs, we examined the transcriptional signatures in cells recovered from animals under each of the viral-exposure conditions (gamma-irradiated 3 dpi, SARS-CoV-2 3 dpi, and SARS-CoV-2 10

21 dpi). To this end, we developed a method in which each population of cells is examined for a

22 clustering bias in a pair-wise comparison along the various significant principal components. We

23 then used the principal component information to determine gene signatures driving the

24 differences. Using this method, we found that macrophages showed large clustering biases in

25 all the comparisons suggesting they had the biggest transcriptional shift over the course of 
1 infection (Fig. S5). The 10 dpi samples, likely due to increases in cell numbers as described

2 above, also showed changes in plasma cells and pneumocytes (Fig. S3 and Fig. S5).

The macrophage analysis was extended by selecting and re-analyzing cells that had either a macrophage or monocyte-like phenotype and further classified to cells with a more tissue-resident phenotype (i.e. alveolar macrophages) or more monocyte-derived phenotype (interstitial macrophages and monocytes) using the expression of MARCO gene (Figure 4A). A comparison of the percentage of the MARCO+/MARCO- cells showed that at $3 \mathrm{dpi}$ there was a large influx of monocyte-derived cells as most of the macrophages detected at that time are MARCO-. This shift began to normalize by $10 \mathrm{dpi}$ as the animals recovered (Fig. 4B). To identify the major pathways whose genes are responsible for the analytical differences between these two cell populations, we performed a similar sub-cluster analysis on the MARCO+/MARCO- cells. For the MARCO+ cells, there was a transcriptional shift at $10 \mathrm{dpi}$ along principal component 1 (PC1) (Fig. 4C). Pro-phagocytic gene sets were enriched along this component, suggesting these macrophages were still active in clearing cell debris from the infected lungs potentially explaining why the macrophages stain for viral antigen at this timepoint (Fig. 2I). Comparatively, along PC2, we see a shift mostly at $3 \mathrm{dpi}$, and only a slight shift at $10 \mathrm{dpi}$. The genes associated with this component are enriched for pro-inflammatory genes such as interferon-stimulated genes (ISGs) (Fig. 4C). In distinction to the MARCO+ cells, for MARCO- cells, along PC1 we saw a shift in the samples at $3 \mathrm{dpi}$, mostly with proinflammatory genes (Fig. 4D). Along PC2 in the MARCO- cells, we see again the pro-

21 phagocytosis genes associated with the endosomes, as well as migratory genes (CD63). This 22 was observed both at 3 and $10 \mathrm{dpi}$ (Fig. 4D), suggesting that at $3 \mathrm{dpi}$, the macrophages are in 23 an inflammatory state that is beginning to resolve by $10 \mathrm{dpi}$, and that the MARCO+ 24 macrophages do not increase their expression of pro-phagocytic genes until later in infection. To assess interactions between macrophages and other cell types, we looked at a 26 combination of the cluster level identity as compared to the individual cell level identity. Using 
1 the same cell identity algorithm, and the clusters calculated by Seurat (Stuart et al., 2019) we

2 looked within individual clusters to identify which cells had a strong macrophage gene marker.

3 At 3 dpi, the clusters associated with pneumocytes, fibroblasts, and endothelial cells all had

4 strong gene markers for macrophages, suggesting these cells were being actively

5 phagocytosed by the macrophages (Fig. 4E). This signature was mostly absent by $10 \mathrm{dpi}$,

6 possibly because the MARCO+ macrophages are picking up more dead cells and cellular debris

7 by this time rather than whole cells with replicating virus (Fig. 4F).

\section{Mediastinal lymph nodes are in an inflammatory state at $3 \mathrm{dpi}$}

To relate these virus replication and inflammatory changes in the lungs to changes in secondary lymphoid tissues, we sampled cells from the mediastinal lymph nodes. We collected whole lymph nodes from animals at the time of necropsy and prepped the samples along with the lung tissue. In the lymph nodes, we were not able to detect any viral RNA by single-cell RNA sequencing, despite a few mononuclear cells staining for viral antigen by IHC (Fig. S2E). This could be due to low abundance of RNA, low sensitivity of the scRNA-Seq assay in its ability to detect lowly abundant transcripts, or an inability to capture high abundance of non-lymphocyte populations from the lymph node when generating cell suspensions (Gerner et al., 2012). Using an annotation strategy like that used for lung cells, we could detect most of the major cell populations of the lymph node, with most cells annotating as T or B lymphocytes (Fig. 5A). Again, as with the lung, the annotated cell types expressed known marker genes associated with their phenotype (Fig. 5B). The largest transcriptional change observed across all the cell

22 types was an increase in interferon responsive genes at 3 dpi suggesting an inflammatory state

23 in the draining lymph nodes. This transcriptional profile was resolved by $10 \mathrm{dpi}$ and was absent

24 in the lymph nodes from animals that received gamma-irradiated virus (Fig. 5C).

There was an increase in the percentage of dividing cells (cells identified as in G2M or 
1 This is consistent with histology findings of lymph node hyperplasia later at $10 \mathrm{dpi}$ (Fig. S2D).

2 Additional changes in cell populations included an increase in plasma cells and plasmablasts by

$310 \mathrm{dpi}$ (Fig. 5E). Interestingly, the latter was also seen in the animals that received the gamma-

$4 \quad$ irradiated virus at $3 \mathrm{dpi}$ (Fig. 5F) suggesting the development of an adaptive immune response

5 to the virus. The development of plasmablasts at 3 dpi in animals that received SARS-CoV-2 is

6 lacking, potentially due to the inflammatory state of the lymph node. When looking at which cell

7 type showed the strongest transcriptional changes, we found that even at $10 \mathrm{dpi}$, macrophages

8 showed the strongest transcriptional shift when compared to samples from animals inoculated

9 with gamma-irradiated virus (Fig. S6A). Within the macrophage population, we saw an

10 upregulation in a small subset of macrophages with markers for fully mature monocyte-derived

11 macrophages such as CHIT1 at $10 \mathrm{dpi}$ (Fig. S6B-C).

Discussion

This study used large scale single cell sequencing in a non-human primate model of SARS-CoV-2 infection. The benefit of using animal models to study SARS-CoV-2 lies in the ability to collect time-resolved datasets of the lungs instead of being limited to sampling at terminal timepoints (Menter et al., 2020; Tian et al., 2020). Using traditional virological methods as well as single-cell sequencing we have begun to parse out the infection dynamics which occur as the disease progresses and virus is eventually cleared in the African green monkey model of mild COVID-19.

One informative aspect of this study was the inclusion of two animals inoculated with gamma-irradiated SARS-CoV-2 which renders the virus unable to replicate (Feldmann et al., 2019). This enabled us to directly compare the detection of viral genomic RNA from replicating vs. non-replicating virus. We found that sgRNA, though present at high levels in the inoculum, was not present in the swab samples collected at 1 and 3 dpi despite evidence of gRNA from 26 the gamma-irradiated virus, indicating that sgRNA is less stable than gRNA and will degrade 
1 quickly without replicating virus generating more such RNA. Such viral dynamics could be due

2 to gRNA being protected and encapsulated or secondary structures which help prevent

3 degradation (Wakida et al., 2020). This has potential implications in patient testing as positive

4 gRNA results by PCR may not represent replication-competent SARS-CoV-2, especially since

5 the highest amount of gRNA detected in the samples from the animals inoculated with irradiated

6 virus were found in the nasal swabs currently used for most patient diagnostics. Our data

7 suggests that PCR-based tests which specifically target SARS-CoV-2 sgRNA may provide a

8 more realistic signature of replicating virus than detection of gRNA, improving models to

9 determine patient infectivity (Bullard et al., 2020; Huang et al., 2020). Since animals cleared

10 sgRNA faster than gRNA the duration of time PCR-positive patients needs to remain in isolation could potentially be reduced. infection at the time of peak disease in the lungs where most virus replication is occurring. Due to the nature of the $10 \mathrm{X}$ genomics platform and the poly-adenylation of the SARS-CoV-2 genome and sgRNA, we were able to determine which cell types were positive for viral RNA in a method similar to the one used by (Bost et al., 2020), though we did not include any other

17 alignments beyond SARS-CoV-2. Importantly, we were able to detect read enrichment across the whole genome of the virus, though the strongest enrichment was at the most 3' end of the genome, followed by the 5' leader TRS. This is consistent with the presence of both gRNA and sgRNA in the lungs at $3 \mathrm{dpi}$. Further investigation into the distribution of reads across the

21 genome suggests that productive virus replication is mostly occurring in infected pneumocytes,

22 though the macrophage population contained the highest percentage of cells positive for viral

23 RNA. A potential reason that the macrophages could have such high viral RNA and

24 immunoreactivity is through phagocytosis of virus particles or infected cells or through abortive

25 replication. Interestingly, abortive replication could result in the production of aberrant replication 
1 products that induce a proinflammatory response as has been shown for influenza $A$ virus in the

2 lungs (Brandes et al., 2013; Te Velthuis et al., 2018).

In the conditions examined here, macrophages appeared to be the major drivers of

4 inflammation in the lungs. This is especially interesting as it has been suggested through the

5 study of healthy human tissue that macrophages interact with ACE2 expressing cells at higher

6 frequencies than other populations (Qi et al., 2020). Both the resident and monocyte-like

7 macrophages at 3 dpi were enriched for pro-inflammatory genes likely caused by having higher

8 titers of virus in the lungs driving the inflammatory state. Interestingly, the MARCO+, or tissue-

9 resident, and MARCO-, or monocyte-derived, macrophage populations showed distinct patterns of gene expression in the 3 and 10 dpi samples. The MARCO+ cells were enriched for ISGs at

$113 \mathrm{dpi}$, but enriched in pathways associated with the endosomes/lysosomes which are up-

12 regulated during a pro-phagocytic period (A-Gonzalez et al., 2017) only at 10 dpi. Only the

13 MARCO- cells at 3 dpi were enriched in genes associated with migration and

endosomes/lysosomes, suggesting that, at $3 \mathrm{dpi}$, macrophages have very different responses to infection based on the cell origin (tissue-resident or recruited) and are likely performing different roles during infection. Further investigation into which macrophage populations are responsible

17 for innate immune host defense and for clearing up the lung environment of cellular and other debris at different stages post-inoculation may help lead to unravel the causes of lung cell damage and indicate potential targets for therapeutic intervention. Interferon stimulated genes (ISGs) represented dominant responses in

21 monocyte/macrophage populations in both the lung and lymph nodes in the early stages of

22 infection but are reduced by the time infection is cleared. In contrast to the AGM model, the role

23 of the IFN response has been proposed as a driver of disease if it is induced with delayed

24 kinetics relative to peak virus replication (Channappanavar et al., 2016; Channappanavar et al., 25 2019). Single-cell approaches in human PBMCs and BAL from patients stratified by COVID-19 26 severity have revealed that type I, II and III IFN is sustained at higher levels in severe patients, 
1 while IFNs declined in moderate patients over time from symptom onset (Liao et al., 2020;

2 Lucas et al., 2020; Wilk et al., 2020). In the AGM model, monocyte recruitment and ISG

3 responses were temporally controlled along with virus replication and were diminished in the

4 lungs during recovery and not sustained in the lymph nodes beyond the stage of peak virus

5 replication. Therefore, this model reflects effective viral control and reveals transcriptional

6 signatures within tissues associated with protective responses.

7 Together, the data reported here provide unique insights into the dynamics of SARS-

8 CoV-2 infection over time in the lungs and associated secondary lymphoid tissue, the identity of

9 the cells hosting replicating virus, and the transcriptional changes in these and other cells during

10 this infectious process. Although the AGM model did not result in severe disease upon

11 inoculation with SARS-CoV-2, it did allow for a deeper understanding of virus replication and

12 host response dynamics along with gene signatures associated with a successful resolution of

13 infection which can be used to inform development of host-targeted therapeutics.

Acknowledgements

16 The authors would like to thank Greg Saturday, Dana Scott, Chad Clancy and all RMVB animal

17 caretakers for help with the animal experiment, Anita Mora for help with figure preparations and

18 Ronald N. Germain for helpful discussions. This work is supported by the Intramural Research

19 Program of NIAID, NIH.

20 Author contributions

21 Conceptualization, E.S., V.J.M., H.F., S.M.B and E.d.W.; investigation E.S., B.N.W., J.L., F.F.,

22 G.S., L.P.-P., K.M.-W., B.J.S., C.M., A.O., C.S. and E.d.W.; writing-original draft, E.S. and

23 E.d.W.; writing, review and editing, all authors.

\section{Declaration of interests}

25 The authors have no competing interests to declare. 


\section{Methods}

\section{Ethics and biosafety statement}

4 All animal experiments were approved by the Institutional Animal Care and Use Committee of

5 Rocky Mountain Laboratories, NIH and carried out by certified staff in an Association for

6 Assessment and Accreditation of Laboratory Animal Care (AAALAC) International accredited

7 facility, according to the institution's guidelines for animal use, following the guidelines and basic

8 principles in the NIH Guide for the Care and Use of Laboratory Animals, the Animal Welfare Act,

9 United States Department of Agriculture and the United States Public Health Service Policy on

10 Humane Care and Use of Laboratory Animals. African green monkeys were housed in adjacent

11 individual primate cages allowing social interactions, in a climate-controlled room with a fixed

12 light-dark cycle (12-hr light/12-hr dark). Animals were monitored at least twice daily throughout

13 the experiment. Commercial monkey chow, treats, and fruit were provided twice daily by trained

14 personnel. Water was available ad libitum. Environmental enrichment consisted of a variety of

15 human interaction, manipulanda, commercial toys, videos, and music. The Institutional

16 Biosafety Committee (IBC) approved work with infectious SARS-CoV-2 strains under BSL3

17 conditions. Sample inactivation was performed according to IBC-approved standard operating

18 procedures for removal of specimens from high containment.

\section{Study design}

20 To evaluate the pathogenesis of SARS-CoV-2 in African green monkeys, eight adult African

21 green monkeys (4 males, and 4 females, body weight $3.5-6 \mathrm{~kg}$ ) were inoculated via a

22 combination of intranasal $(0.5 \mathrm{ml}$ per nostril), intratracheal $(4 \mathrm{ml})$, oral $(1 \mathrm{ml})$ and ocular $(0.25 \mathrm{ml}$

23 per eye) of a $4 \times 10^{5} \mathrm{TCID} 50 / \mathrm{ml}\left(3 \times 10^{8}\right.$ genome copies $\left./ \mathrm{ml}\right)$ virus dilution in sterile DMEM. Two

24 control animals ( 1 male and 1 female, body weight $4.5-5.5 \mathrm{~kg}$ ) were inoculated via the same

25 routes with the same dose and volume of inoculum that was gamma-irradiated to render the

26 virus noninfectious (Feldmann et al., 2019). The animals were observed twice daily for clinical 
1 signs of disease using a standardized scoring sheet (Munster et al., 2020); the same person

2 assessed the animals throughout the study. The predetermined endpoint for this experiment

3 was 3 days post inoculation (dpi) for the two control animals that were inoculated with irradiated

4 virus and one group of 4 animals inoculated with infectious SARS-CoV-2, and 10 dpi for the

5 remaining 4 animals inoculated with infectious SARS-CoV-2. Clinical exams were performed on

$6 \quad 0,1,3,5,7$, and 10 dpi on anaesthetized animals. On exam days, clinical parameters such as

7 bodyweight, body temperature and respiration rate were collected, as well as ventro-dorsal and

8 lateral chest radiographs. Blood and nasal, throat, and rectal swabs were collected during all

9 clinical exams. Additionally, during on 1, 3, and 5 dpi animals were intubated and

10 bronchoalveolar lavages were performed using $10-20 \mathrm{ml}$ sterile saline. After euthanasia,

11 necropsies were performed. The percentage of gross lung lesions was scored by a board-

12 certified veterinary pathologist and samples of the following tissues were collected: cervical

13 lymph node, conjunctiva, nasal mucosa, nasal septum, oropharynx, tonsil, trachea, all six lung

14 lobes, mediastinal lymph node, right and left bronchus, heart, liver, spleen, kidney, stomach,

15 duodenum, jejunum, ileum, cecum, colon, and urinary bladder. Histopathological analysis of

16 tissue slides was performed by a board-certified veterinary pathologist blinded to the group

17 assignment of the animals.

18 Virus and cells

19 SARS-CoV-2 isolate nCoV-WA1-2020 (MN985325.1) (Harcourt et al., 2020) (Vero passage 3)

20 was kindly provided by CDC and propagated once in VeroE6 cells in DMEM (Sigma)

21 supplemented with $2 \%$ fetal bovine serum (Gibco), $1 \mathrm{mM} \mathrm{L-glutamine} \mathrm{(Gibco),} 50 \mathrm{U} / \mathrm{ml}$ penicillin

22 and $50 \mu \mathrm{g} / \mathrm{ml}$ streptomycin (Gibco) (virus isolation medium). The used virus stock was $100 \%$

23 identical to the initial deposited genbank sequence (MN985325.1) and no contaminants were

24 detected. This virus was gamma-irradiated with a dose of 2MRad to produce a non-infectious

25 inoculum (Feldmann et al., 2019). Absence of infectious virus after gamma-irradiation was 
1 confirmed in Vero E6 cells. VeroE6 cells were maintained in DMEM supplemented with $10 \%$

2 fetal calf serum, $1 \mathrm{mM} \mathrm{L}$-glutamine, $50 \mathrm{U} / \mathrm{ml}$ penicillin and $50 \mu \mathrm{g} / \mathrm{ml}$ streptomycin.

3 Quantitative PCR

$4 \quad$ RNA was extracted from swabs and BAL using the QiaAmp Viral RNA kit (Qiagen) according to

5 the manufacturer's instructions. Tissues ( $30 \mathrm{mg}$ ) were homogenized in RLT buffer and RNA was

6 extracted using the RNeasy kit (Qiagen) according to the manufacturer's instructions. For

7 detection of genomic and subgenomic RNA, $5 \mu$ RNA was used in a one-step real-time RT-

8 PCR assay (Corman et al., 2020; Wolfel et al., 2020) using the Rotor-Gene probe kit (Qiagen)

9 according to instructions of the manufacturer. In each run, standard dilutions of counted RNA

10 standards were run in parallel, to calculate copy numbers in the samples.

\section{Virus titration and isolation}

12 Virus titrations were performed by end-point titration in Vero E6 cells. Cells were inoculated with

1310 -fold serial dilutions of swab and BAL samples. Virus isolation was performed on tissues by

14 homogenizing the tissue in $1 \mathrm{ml}$ DMEM and inoculating Vero E6 cells in a 24 well plate with 250

$15 \mu \mathrm{l}$ of cleared homogenate and a 1:10 dilution thereof. One hour after inoculation of cells, the

16 inoculum was removed and replaced with $100 \mu \mathrm{l}$ (virus titration) or $500 \mu \mathrm{l}$ virus isolation

17 medium. Six days after inoculation, CPE was scored and the TCID50 was calculated.

18 Histopathology

19 Histopathology, immunohistochemistry and in situ hybridization were performed on African

20 green monkey tissues. After fixation for a minimum of 7 days in $10 \%$ neutral-buffered formalin

21 and embedding in paraffin, tissue sections were stained with hematoxylin and eosin (HE).

22 Immunohistochemistry was performed using a custom-made rabbit antiserum against SARS-

$23 \mathrm{CoV}-2 \mathrm{~N}$ at a 1:1000 dilution, using a CD68 clone KP1 mouse monoclonal antibody

24 (Dako/Agilent cat \#M0814) at a 1:100 dilution to identify macrophages, and using Cytokeratin

25 clone AE1/AE3 mouse monoclonal antibody (Dako/Agilent cat \#M3515) at a 1:100 dilution to

26 identify epithelial cells. In situ hybridization was used for detection of SARS-CoV-2 RNA in 
1 selected whole tissue sections of the lungs using the RNAscope VS Universal AP assay

2 (Advanced Cell Diagnostics Inc.) as described previously (de Wit et al., 2013) and using probe

3 directed against the SARS-CoV-2 S gene (cat\# 848569). Stained slides were analyzed by a

4 board-certified veterinary pathologist.

\section{Single-Cell Sequencing of lung and mediastinal lymph nodes}

6 Lung sections and mediastinal lymph nodes were taken at the time of necropsy of the animals

7 and processed. Cell suspensions were generated by manually dicing tissue, enzymatically

8 digesting in RPMl containing $0.1 \mathrm{mg} / \mathrm{ml}$ Liberase TM (Sigma 5401127001 ) and $0.02 \mathrm{mg} / \mathrm{ml}$

9 DNasel (Sigma 11284932001 ) at $37^{\circ} \mathrm{C}$, and then passing through a 100 um filter. Suspensions were subjected to ACK lysis and final washes in PBS containing 0.1\% MACS BSA (Miltenyi 130-091-386). 10,000 cells were prepared for $10 \mathrm{X}$ Genomics gel bead emulsions. The 10X genomics version 3.0 chemistry was used. cDNA for the individual cells was generated and libraries prepped according to the manufactures protocol. After the final libraries were generated, samples were inactivated for any potentially remaining virus using 500ul of AVL with $500 u$ of ethanol with a sample volume of $140 u$ u. After a minimum 10-minute incubation, samples were removed from the high-containment laboratory following standard protocols and the libraries were extracted from the AVL using the Qiagen AllPrep DNA spin columns (Cat 80204). Samples were then sent for quantification and sequencing. Samples were sequenced on the NextSeq550 using the 10X suggested cycling.

Processing of single-cell sequencing samples

21 Samples were process through the cellRanger pipeline to perform de-multiplexing and generate

22 count tables. Alignment was done against the African Green Monkey Ensembl genome

23 (ChISab1.1) with the SARS-CoV-2 genome (NC_045512.2) included to be able to parse out

24 reads associated with the viral genome. Samples were then read into R (V3.6.2) using Seurat

25 (V3.1.5) (Stuart et al., 2019). Since samples were collected across two different days (day 3 and 26 day 10 post infection), we wanted to account for potential batch effects in the global dataset. For 
1 this reason, we then integrated the samples using the IntegrateData function. Cells were filtered

2 that contained abnormally high mitochondrial genes (greater than 3 standard deviations above

3 the median) and cells that are likely doublets were re-labeled (ratio of unique features to UMI

4 per cell $<0.15$ ). Also, cells containing less than or greater than $3 \mathrm{sd}$ of UMI compared to the

5 population total were removed to filter for noise. Finally, the PCA and UMAP projections were

6 calculated for the samples and significant clusters of cells were identified. The lung and the

7 lymph node samples were analyzed separately. Gene set enrichment analysis was performed

8 using fgsea (Sergushichev, 2016) and the MSig DB (Subramanian et al., 2005) c2cp gene sets.

9 Cell type identification for single-cell sequencing data

10 To determine the identity of either clusters of cells or individual cells, we developed an unbiased

11 method that uses a transcriptional profile of cells instead a few known marker genes. For the

12 reference data, we used an annotated single cell sequencing dataset from (Madissoon et al.,

13 2019). For each of the cell type present in the dataset of lung or spleen tissue, we calculated the

14 differential gene expression using the FindMarkers function in Seurat. To find genes strongly associated with each individual cell type we filtered the data to contain only those genes with an average $\log F G$ greater than 1 and where the difference in the percentage of cells in the cell type

17 of interest expressing the gene compared to the percentage of cells in all other cell types expressing the gene was greater than 0.5 . We used this gene set in either the lung or spleen of the human samples to develop the marker gene set and calculated the average expression of the marker gene set in each cell type. This generated a matrix of the marker genes to cell types.

21 Then a correlation of the marker genes in the annotated data was compared to the individual

22 cell or cluster in the African green monkey data. This generated a score for the unknown cell or

23 cluster to a known annotation. Using this method, we found that most clusters contained

24 predominately just 1 cell type (Fig. 4E,F). This is a similar method that was developed for the

25 mouse cell atlas (Han et al., 2018). The results were validated by looking at the expression of

26 the marker genes across the different cell annotations (Fig. 3B and Fig. 5B). When determining 
1 the identity on an individual cell level, an additional step was added to help correct falsely

2 identified cells. Using the knn graph generated in the FindNeighbors function in Seurat, for each

3 cell, its closest neighbors were determined. Once the nearest neighbors were determined, the

4 identities of these neighbors were pulled out. If $>70 \%$ of the nearest neighbors had one specific

5 identity, the cell identity was reassigned as such. This was run multiple times until a stable

6 number of unidentified cells was found (determined by small changes in unknown cell

7 identities). For those that were not able to be identified, the identity from the original

8 transcriptional profile was used.

$9 \quad$ Finally, to identify clusters that are specifically cells undergoing rapid cell division, we

10 used the CellCycleScore function in Seurat to identify which cell cycle each cell was likely in.

11 We then determined that clusters where greater than $95 \%$ of the cells were in G2M or S phase

12 were dividing clusters and were labeled as such.

\section{SARS-CoV-2 read enrichment}

14 To analyze the enrichment of reads across the SARS-CoV-2 genome, we used Integrative

15 Genome Viewer (Robinson et al., 2011) to find read pile-ups. Cells were labeled as positive for

16 viral RNA if they contained any counts to the viral genome.

\section{Clustering biases in scRNA-Seq Data}

18 To determine if there is a clustering bias between two cell types, a new method was developed.

19 Across any cluster of cells, the dataset is subset and re-normalized internally to that cluster.

20 Then the significant principal components (PC) (containing up to $99 \%$ of the variance explained)

21 are calculated. Along each principal component, the location of the cells is pulled and grouped

22 based on the two conditions that are being compared. The median location of each of the cell

23 populations along the PC is calculated and the distance measured. This is carried out for all

24 PCs and all clusters. To identify outliers with the strongest clustering bias, points outside the

25 mean and 2 standard deviations across all the PCs and cell types are noted.

\section{Statistical Analysis}


1 Statistical tests comparing cell numbers were carried out in Prism V8 using an ANOVA.

2 Statistical tests for gene expression in single cell data was carried in Seurat V3.6. Graphs were

3 generated either in ggplot2 (Wickham, 2016) or Prism V8.

\section{Data availability}

5 Animal model data have been deposited in Figshare:

6 https://doi.org/10.6084/m9.figshare.12818726.v1. RNA sequencing data have been deposited in

7 NCBI's Gene Expression (Edgar et al., 2002) and are accessible through GEO Series accession

8 number GSExxx (https://www.ncbi.nlm.nih.gov/geo/query/acc.cgi?acc=GSExxx).

10 Table and figures

11 Table 1. Clinical signs observed in African green monkeys inoculated with irradiated or 12 infectious SARS-CoV-2.

\begin{tabular}{|c|c|c|c|c|}
\hline Inoculum & Animal & $\begin{array}{l}\text { Clinical signs } \\
\text { observed } 1-3 \text { dpi }\end{array}$ & $\begin{array}{l}\text { Clinical signs } \\
\text { observed 4-10 dpi }\end{array}$ & $\begin{array}{l}\text { Observations at } \\
\text { necropsy }\end{array}$ \\
\hline \multirow{2}{*}{$\begin{array}{l}\text { Irradiated } \\
\text { SARS- } \\
\text { CoV-2 }\end{array}$} & AGM1 & $\begin{array}{l}\text { Reduced appetite. } \\
\text { Euthanized } 3 \text { dpi. }\end{array}$ & $\mathrm{N} / \mathrm{A}$ & None. \\
\hline & AGM2 & $\begin{array}{l}\text { Reduced appetite. } \\
\text { Euthanized } 3 \text { dpi. }\end{array}$ & $\mathrm{N} / \mathrm{A}$ & None. \\
\hline \multirow[t]{6}{*}{$\begin{array}{l}\text { SARS- } \\
\text { CoV-2 }\end{array}$} & AGM3 & $\begin{array}{l}\text { Reduced appetite. } \\
\text { Euthanized } 3 \text { dpi. }\end{array}$ & $\mathrm{N} / \mathrm{A}$ & $\begin{array}{l}\text { Gross lung lesions; } \\
\text { cervical and mediastinal } \\
\text { lymph nodes enlarged. }\end{array}$ \\
\hline & AGM4 & $\begin{array}{l}\text { Tachypnea; } \\
\text { reduced appetite. } \\
\text { Euthanized } 3 \text { dpi. }\end{array}$ & $N / A$ & $\begin{array}{l}\text { Gross lung lesions; } \\
\text { mediastinal lymph } \\
\text { nodes mildly enlarged. }\end{array}$ \\
\hline & AGM5 & $\begin{array}{l}\text { Tachypnea; } \\
\text { reduced appetite. } \\
\text { Euthanized } 3 \text { dpi. }\end{array}$ & $N / A$ & $\begin{array}{l}\text { Gross lung lesions; } \\
\text { mediastinal lymph } \\
\text { nodes enlarged. }\end{array}$ \\
\hline & AGM6 & $\begin{array}{l}\text { Reduced appetite. } \\
\text { Euthanized } 3 \text { dpi. }\end{array}$ & $\mathrm{N} / \mathrm{A}$ & $\begin{array}{l}\text { Gross lung lesions; } \\
\text { mediastinal lymph } \\
\text { nodes enlarged. }\end{array}$ \\
\hline & AGM7 & Reduced appetite. & $\begin{array}{l}\text { Reduced appetite. } \\
\text { Recovered at } 5 \mathrm{dpi} \text {. }\end{array}$ & $\begin{array}{l}\text { Gross lung lesions; } \\
\text { mediastinal lymph } \\
\text { nodes enlarged. }\end{array}$ \\
\hline & AGM8 & $\begin{array}{l}\text { Hunched posture; } \\
\text { tachypnea; } \\
\text { severely reduced } \\
\text { appetite. }\end{array}$ & $\begin{array}{l}\text { Hunched posture; } \\
\text { tachypnea; severely } \\
\text { reduced appetite. } \\
\text { Recovered at } 9 \text { dpi. }\end{array}$ & $\begin{array}{l}\text { Lungs consolidated; } \\
\text { mediastinal lymph } \\
\text { nodes enlarged and } \\
\text { hemorrhagic. }\end{array}$ \\
\hline
\end{tabular}


AGM9 Tachypnea; $\quad$ Tachypnea; reduced Gross lung lesions; reduced appetite. appetite. mediastinal lymph

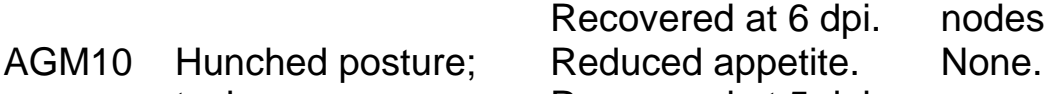
tachypnea; coughing; reduced Recovered at $5 \mathrm{dpi}$. appetite.
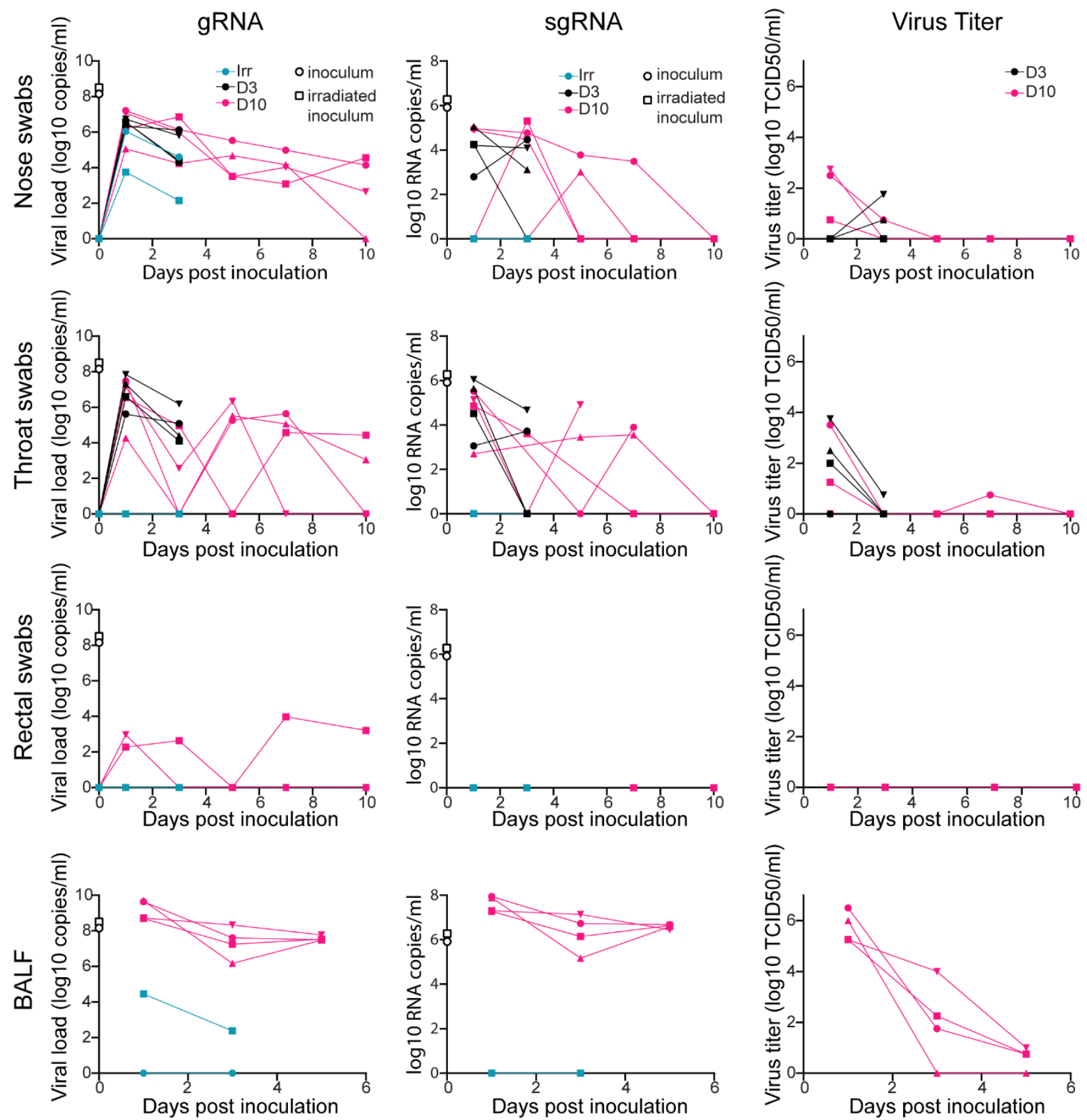
African green monkeys (AGM) were inoculated with gamma-irradiated SARS-CoV-2 $(n=2)$. Eight AGM were inoculated with infectious SARS-CoV-2 isolate nCoV-WA1-2020. After inoculation, 
clinical exams were performed during which nose, throat and rectal swabs were collected; bronchoalveolar lavages (BAL) were performed at 1, 3, and 5 dpi on the four animals remaining in the study through $10 \mathrm{dpi}$ and viral loads and titers were measured. qRT-PCR was performed to detect genomic (left column) and subgenomic RNA (middle column), and in vitro virus titration was performed to detect levels of infectious virus (right column) in these samples. Amount of gRNA and sgRNA in the inocula (gamma-irradiated and infectious) is indicated at timepoint zero. Teal: animals inoculated with gamma-irradiated virus; black: animals inoculated with infectious virus and euthanized at $3 \mathrm{dpi}$; pink: animals inoculated with infectious virus and euthanized at $10 \mathrm{dpi}$. Where indicated, identical symbols have been used to identify individual animals throughout the figures in this manuscript.

Irradiated Control
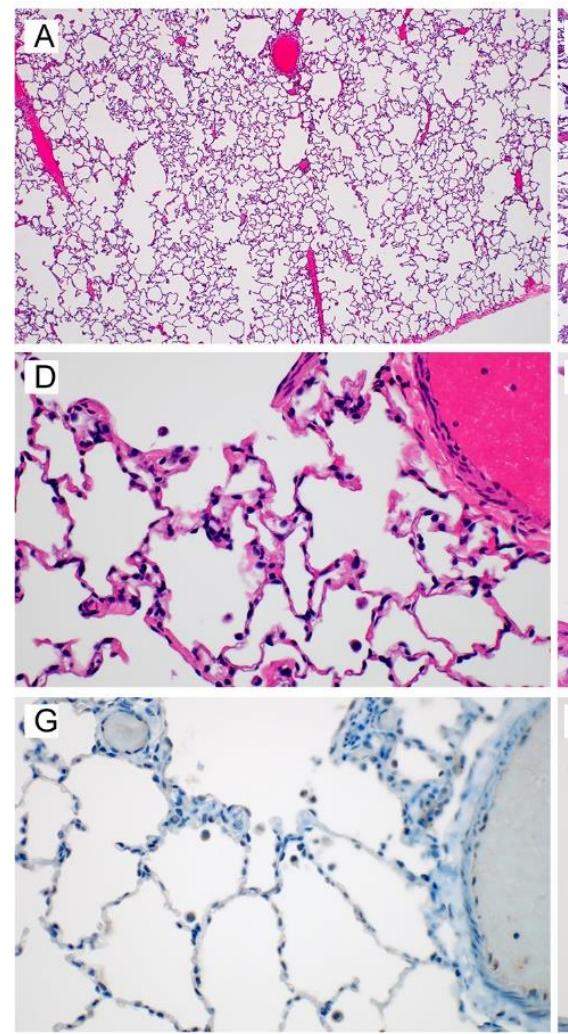

D3

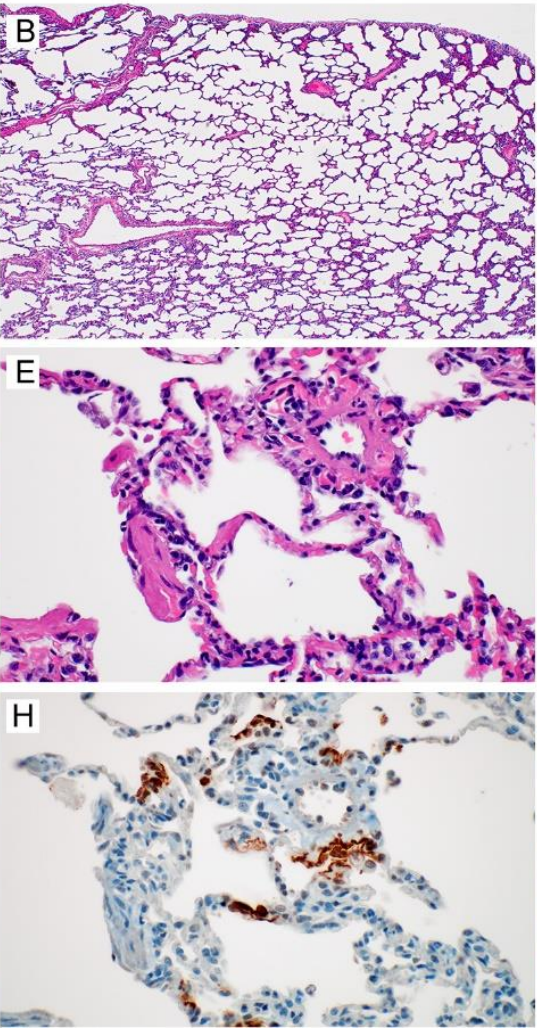

D10

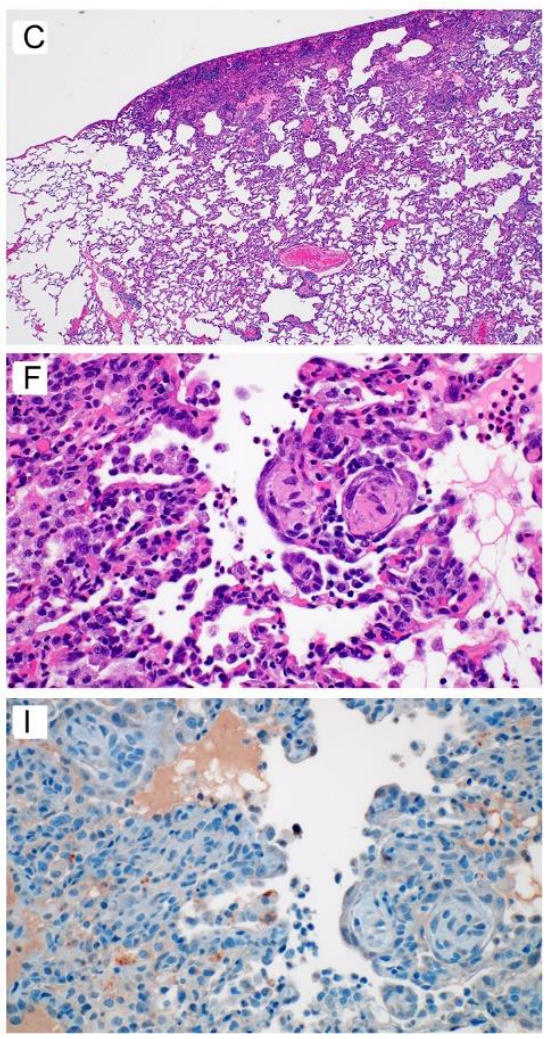

Figure 2. Histological changes in the lungs of African green monkeys inoculated with SARS-CoV-2. African green monkeys were inoculated with gamma-irradiated SARS-CoV-2 $(n=2)$ and euthanized at $3 \mathrm{dpi}$; eight animals were inoculated with SARS-CoV-2 isolate nCoVWA1-2020 and four of those were euthanized at $3 \mathrm{dpi}$, and four at $10 \mathrm{dpi}$. Histological analysis was performed on lung tissue from all animals. (a) Lungs of animals inoculated with gammairradiated SARS-CoV-2 were normal at 3 dpi. (b) Mildly thickened septa were observed at 3 dpi in animals inoculated with infectious SARS-CoV-2. (c) Discrete focus of interstitial pneumonia at the periphery of the lung at $10 \mathrm{dpi}$. (d) High magnification of normal lung tissue in (a). (e) Alveolar septa are slightly thickened and more cellular at 3 dpi. (f) Alveolar edema ( ${ }^{*}$ ), type II pneumocyte hyperplasia (arrowhead), increased alveolar macrophages (arrow) and infiltrating lymphocytes and neutrophils are observed at $10 \mathrm{dpi}$, as well as proliferative nodules associated with terminal airways resembling obstructive bronchiolitis (OB). (g) No SARS-CoV-2 antigen could be detected in lungs from animals inoculated with gamma-irradiated SARS-CoV-2. (h) 
bioRxiv preprint doi: https://doi.org/10.1101/2020.08.20.258087; this version posted August 20,2020. The copyright holder for this preprint (which was not certified by peer review) is the author/funder. This article is a US Government work. It is not subject to copyright under 17 USC 105 and is also made available for use under a CCO license.

1 Cytoplasmic and membrane-associated viral antigen in pneumocytes at 3 dpi. (i) Rare viral 2 antigen could be detected in mononuclear cells, presumably alveolar macrophages, with 3 cytoplasmic debris (arrow) at $10 \mathrm{dpi}$; background blush is observed in alveolar proteinaceous 4 fluid $\left(^{*}\right)$, but pneumocytes do not exhibit immunoreactivity (arrowhead). Magnification a-c 40x, d5 i 400x. 
bioRxiv preprint doi: https://doi.org/10.1101/2020.08.20.258087; this version posted August 20,2020. The copyright holder for this preprint (which was not certified by peer review) is the author/funder. This article is a US Government work. It is not subject to copyright under 17 USC 105 and is also made available for use under a CCO license.

\section{5}
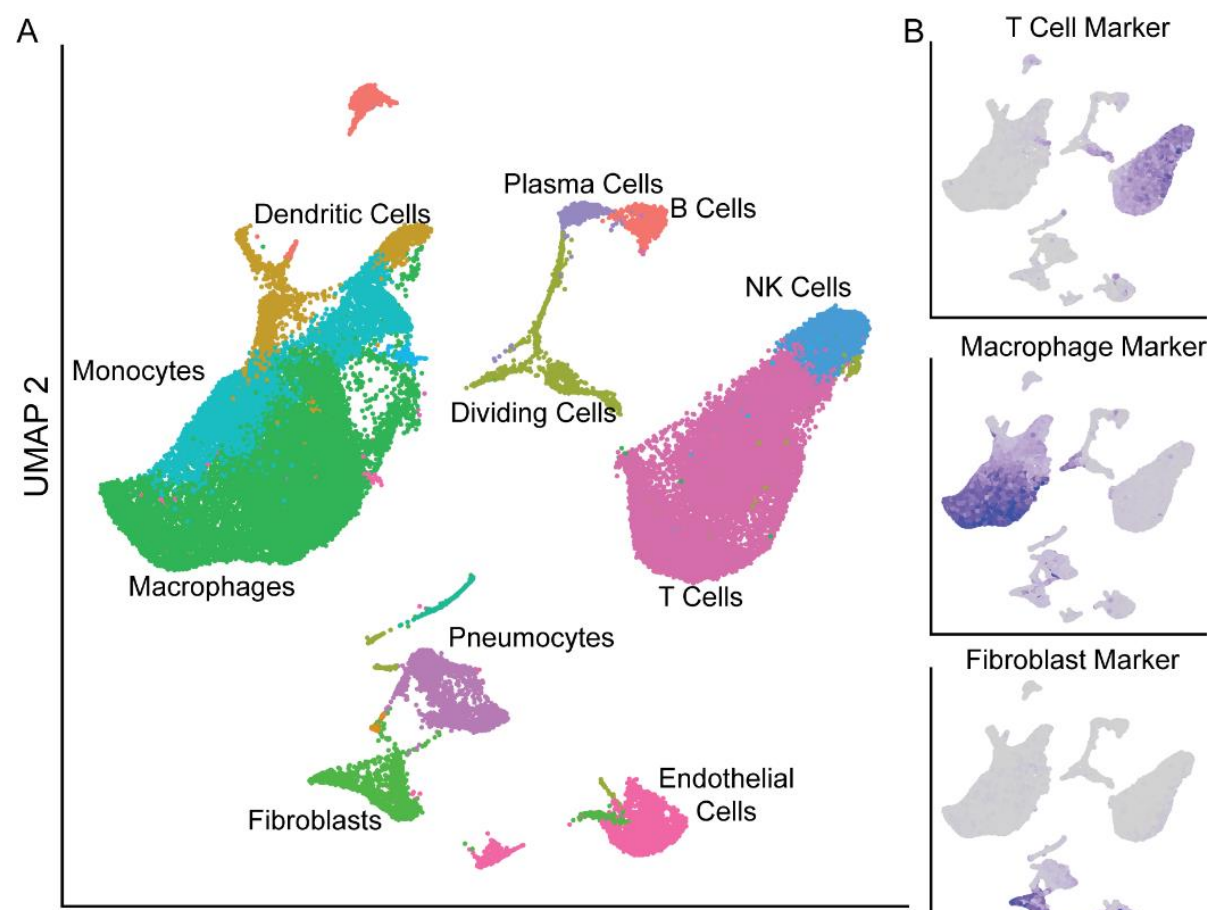

Fibroblast Marker

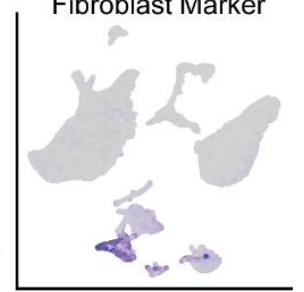

F

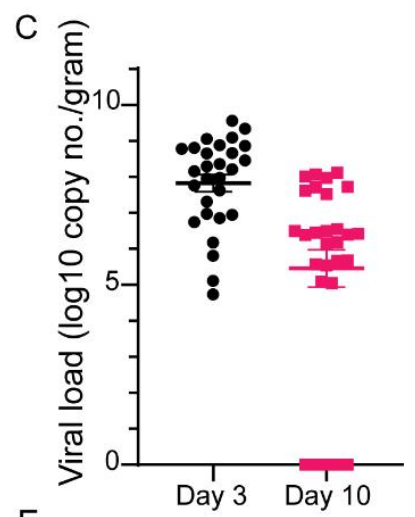

E

D
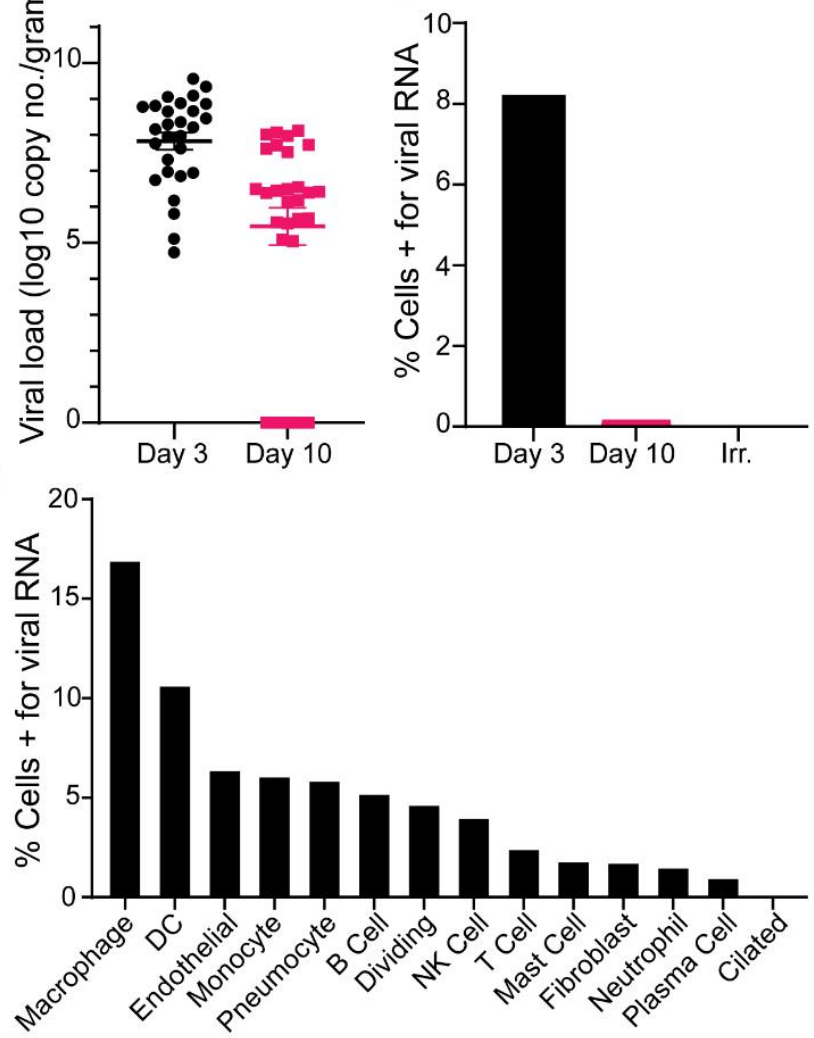

Figure 3. Single cell sequencing and viral dynamics in lung tissue. A. UMAP projection of single-cell RNA sequencing data from whole lung sections from all 10 animals combined. Each 
1 point is an individual cell; colors are based on cell type annotation. Cell names are shown next 2 to their largest cluster. B. Validation of cell type identities using marker gene sets. The darker 3 the purple in each cell the higher its expression of the marker set. Grey means the cell did not 4 express any genes in the marker set. C. Viral load information from the lungs via qRT-PCR for 5 gRNA, grouped for all lobes across all animals. D. Percentage of cells identified by single cell 6 RNA-sequencing that are positive for any reads aligning to the viral genome by days post

7 inoculation. E. Percentage of cells from the 3 dpi samples positive for any reads aligning to the 8 viral genome grouped by cell type. F. The number of cells grouped by cell type (colored to 9 match the UMAP in A) with reads aligning to various other locations across the viral genome, all 10 normalized to the number of cells expressing N. Genes are ordered from the 3' to 5' end of the 11 SARS-CoV-2 genome. G. ISH for viral S RNA in lung tissues at 3 dpi (viral RNA stains red) at 12 100x magnification and 400x magnification. 
bioRxiv preprint doi: https://doi.org/10.1101/2020.08.20.258087; this version posted August 20, 2020. The copyright holder for this preprint (which was not certified by peer review) is the author/funder. This article is a US Government work. It is not subject to copyright under 17 USC 105 and is also made available for use under a CCO license.

A

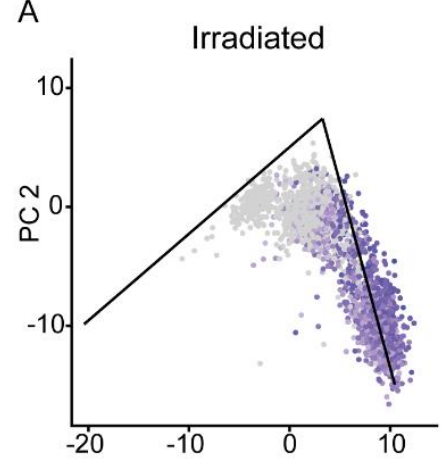

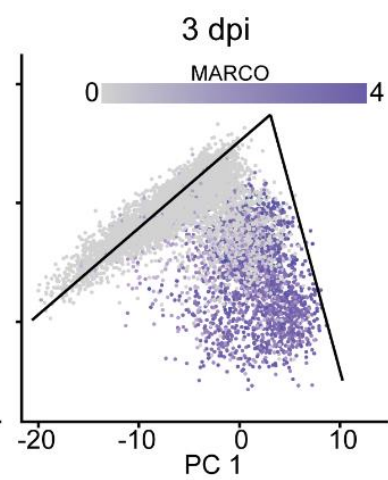

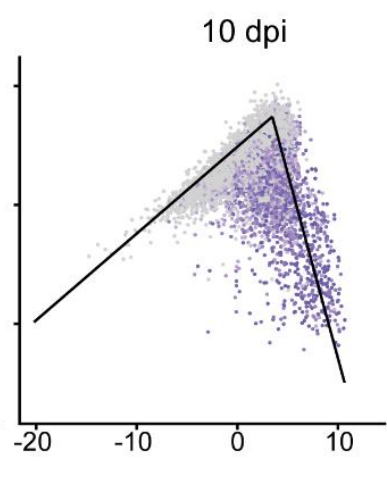

B

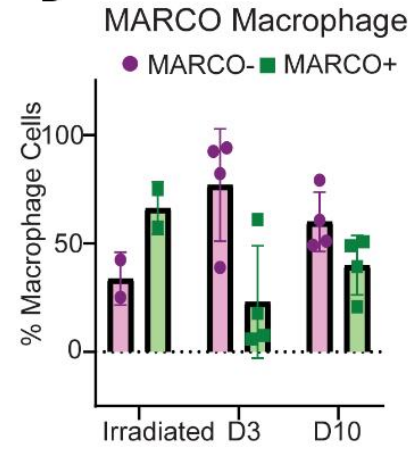

$\circ$
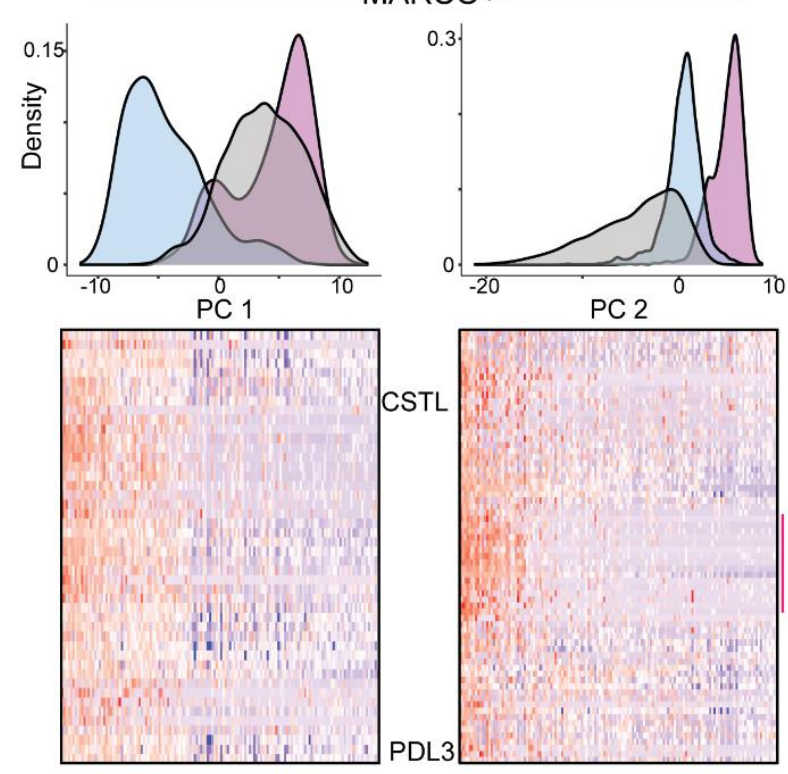

E
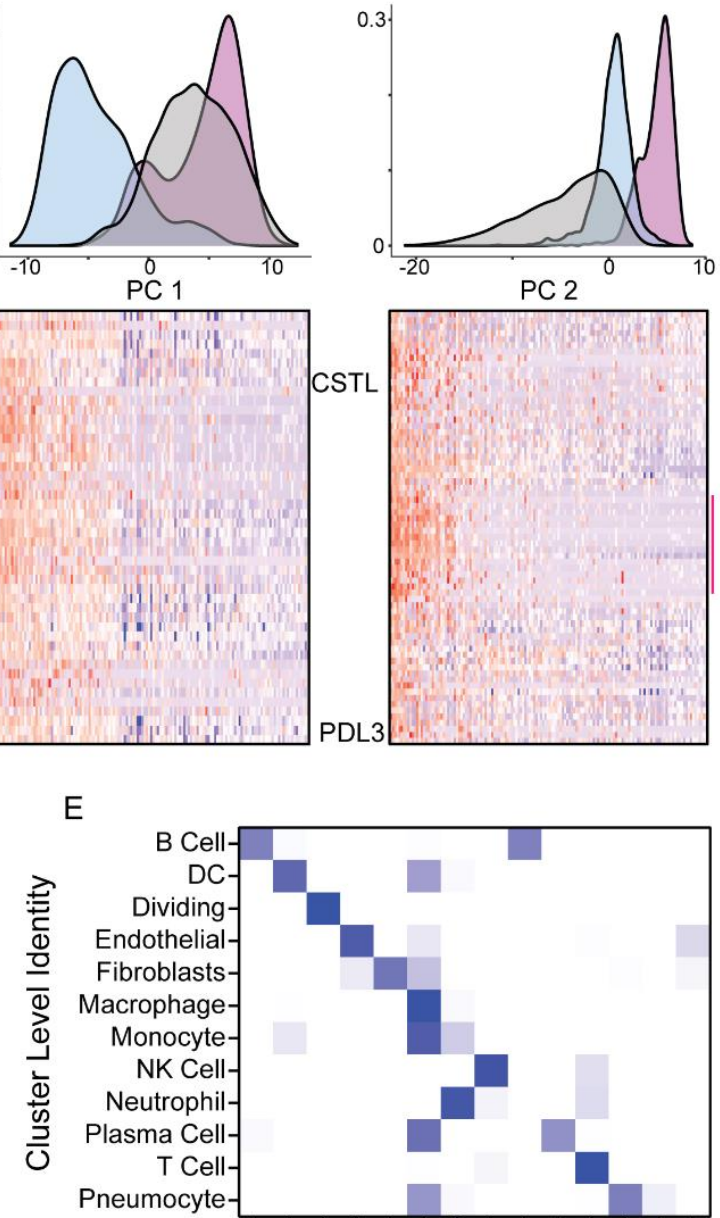
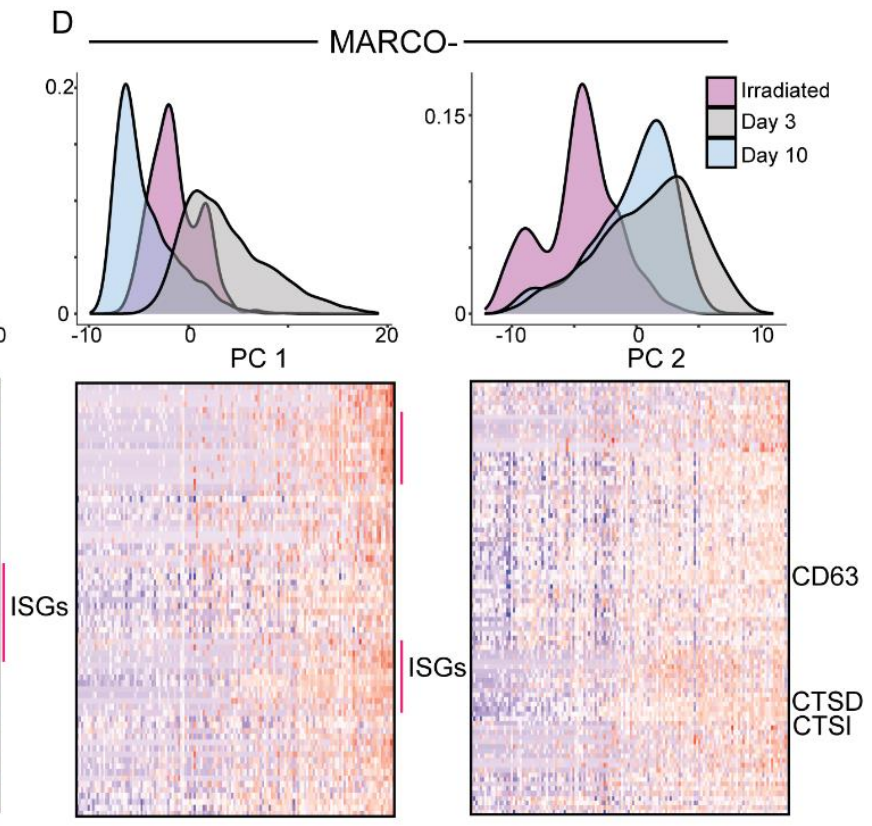

$\mathrm{F}$

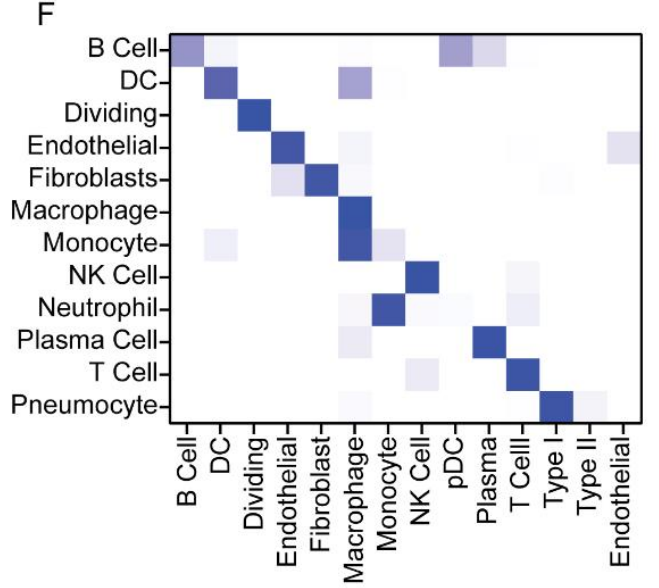

Individual Cell Level Identity

Figure 4. Changes in macrophage populations in the lungs during SARS-CoV-2 infection. A. Principal component analysis of the macrophages. The $x$-axis is pc1 and the $y$-axis is pc2. The graph is split by the experimental groups. Each point is an individual cell and is colored based on the expression of MARCO with grey representing zero expression and the dark purple 
1 represent increased expression. The lines on the PC graphs are for reference across the 2 samples and represent matching locations. B. The percentage of macrophages that are $3 \mathrm{MARCO}+$ (green) or MARCO - (purple) across the three different conditions (x-axis) C. The 4 MARCO+ macrophages principal component analysis (density plots) grouped by the different 5 conditions. Blue: animals inoculated with gamma-irradiated virus and euthanized on 3 dpi; grey: 6 animals inoculated with SARS-CoV-2 and euthanized at 3 dpi; pink: animals inoculated with 7 SARS-CoV-2 and euthanized at $10 \mathrm{dpi}$. The left density plot is pc1 with the heatmap below 8 showing the individual cells (columns) sorted based on their location along pc1 with top genes 9 showing high correlation along that principal component clustered (rows). Darker blue means low to no expression and a dark red color means high expression. A similar graph, but for PC2 is on the right. A few of the gene names are noted just to the right of the heatmaps. D. A similar set of graphs as in panel C but for the MARCO- cells. E. Showing the comparison between the individual cell identity (columns) and the cluster level identity (rows) based on the unbiased identification algorithm at 3 dpi for SARS-CoV-2-infected animals. The colors represent the \% of individual cells in the cluster that match the identified phenotype. White means no individual cell in that cluster match the phenotype; whereas the darker the blue the higher the percentage of matching cells. F. A similar graph to $E$ at 10 dpi for SARS-CoV-2-infected animals. 
A

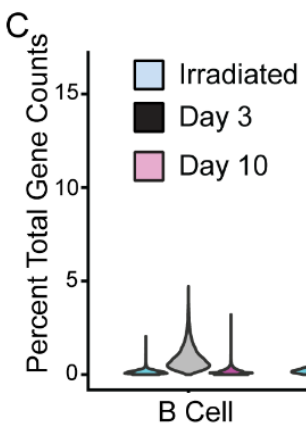

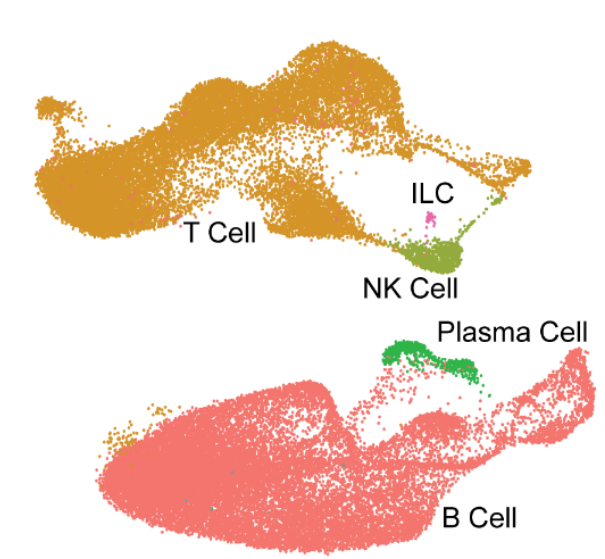

UMAP

\section{$\mathrm{DC}$}

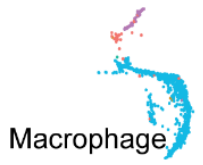

pDC
B

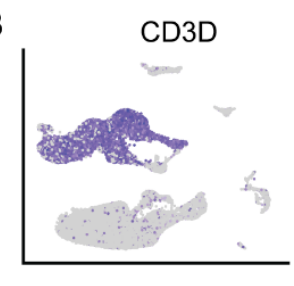

KLRF1

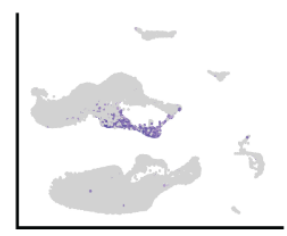

FCER1G

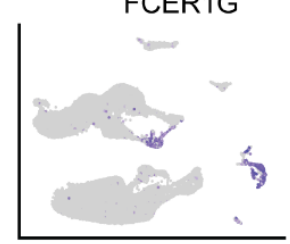

HLA-DRA
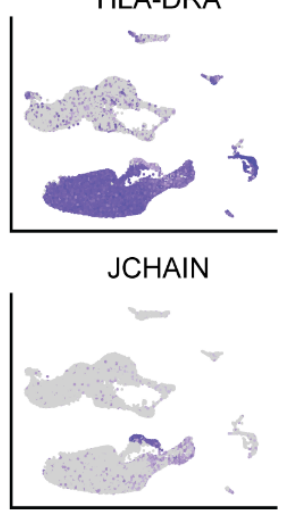

PTPRC

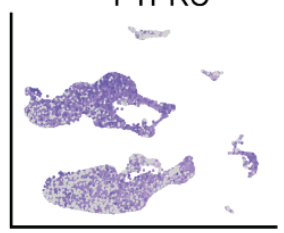

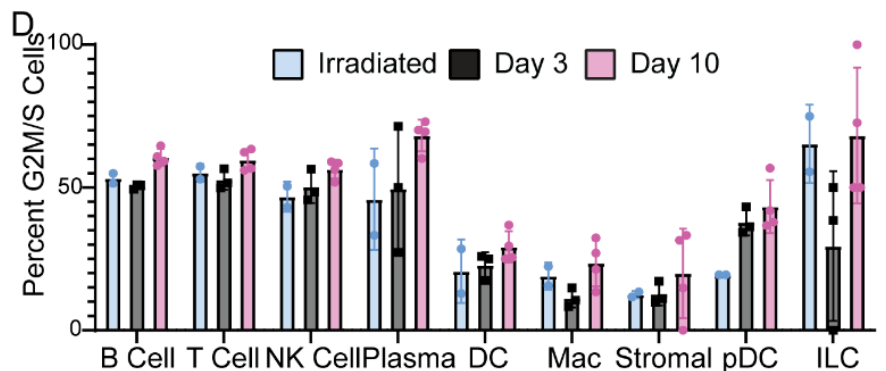

Interferon Responsive Genes
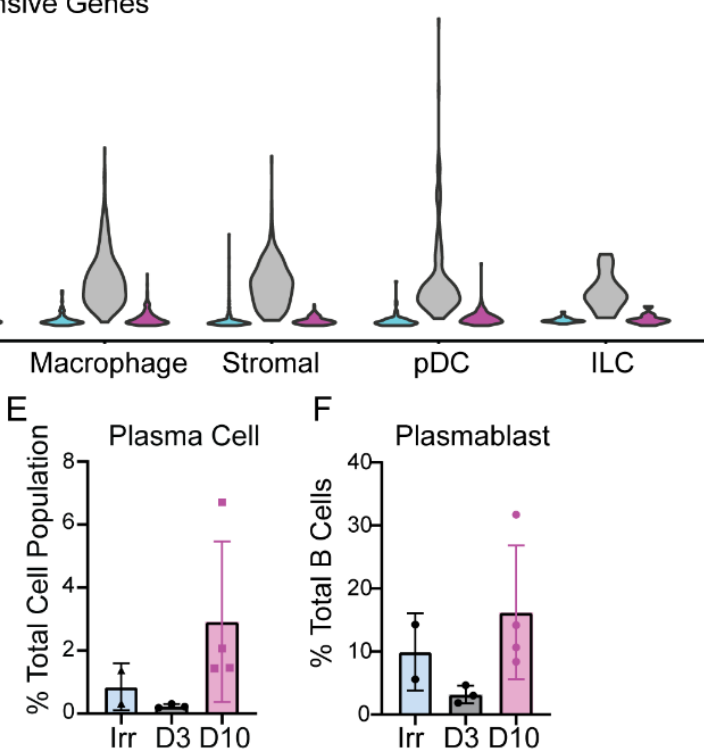

Figure 5. Single cell sequencing of mediastinal lymph nodes. A. UMAP projection of single cell sequencing data from lymph nodes of all 10 animals combined. Each point represents an individual cell and cells are colored based on their cell type. The names of the cell types are placed next to their largest cluster. B. Single gene expression to validate cell type identifications in A. For each graph in this panel, grey means the cell had zero expression and the darker the purple, the higher the expression of that gene. C. percent of total gene counts for each cell for a subset of interferon responsive genes (y-axis). The $x$-axis is split by the cell types and further divided by sample: blue represents animals inoculated with gamma-irradiated virus and euthanized at $3 \mathrm{dpi}$; grey represents animals inoculated with SARS-CoV-2 and euthanized at 3 dpi; pink represents animals inoculated with SARS-CoV-2 and euthanized at $10 \mathrm{dpi}$. Individual cells are removed for clarity and only densities of cells across the populations are shown in violin plots. D. Percentage of each cell population (x-axis) that is actively dividing (stage G2M or 

D., Strong, J.E., Alexander, D., Garnett, L., Boodman, C., Bello, A., Hedley, A., Schiffman, Z., et al.

S) as determined by a profile of gene expression. Each point is an individual animal and bars represent the mean and standard deviation of the samples. E. Percentage of plasma cells in each animal's sample compared it's total cell numbers. F. Percentage of plasmablast cells in each animal's sample compared to the total B cell population.

\section{References}

A-Gonzalez, N., Quintana, J.A., Garcia-Silva, S., Mazariegos, M., Gonzalez de la Aleja, A.,

Nicolas-Avila, J.A., Walter, W., Adrover, J.M., Crainiciuc, G., Kuchroo, V.K., et al. (2017).

Phagocytosis imprints heterogeneity in tissue-resident macrophages. J Exp Med 214, 12811296.

Bost, P., Giladi, A., Liu, Y., Bendjelal, Y., Xu, G., David, E., Blecher-Gonen, R., Cohen, M., Medaglia, C., Li, H., et al. (2020). Host-Viral Infection Maps Reveal Signatures of Severe COVID-19 Patients. Cell 181, 1475-1488 e1412.

Brandes, M., Klauschen, F., Kuchen, S., and Germain, R.N. (2013). A systems analysis identifies a feedforward inflammatory circuit leading to lethal influenza infection. Cell 154, 197212.

samples. Clin Infect Dis. 
1 Channappanavar, R., Fehr, A.R., Vijay, R., Mack, M., Zhao, J., Meyerholz, D.K., and Perlman,

2 S. (2016). Dysregulated Type I Interferon and Inflammatory Monocyte-Macrophage Responses

3 Cause Lethal Pneumonia in SARS-CoV-Infected Mice. Cell Host Microbe 19, 181-193.

4 Channappanavar, R., Fehr, A.R., Zheng, J., Wohlford-Lenane, C., Abrahante, J.E., Mack, M.,

5 Sompallae, R., McCray, P.B., Jr., Meyerholz, D.K., and Perlman, S. (2019). IFN-I response

6 timing relative to virus replication determines MERS coronavirus infection outcomes. J Clin

$7 \quad$ Invest 129, 3625-3639.

8 Chua, R.L., Lukassen, S., Trump, S., Hennig, B.P., Wendisch, D., Pott, F., Debnath, O.,

9 Thurmann, L., Kurth, F., Volker, M.T., et al. (2020). COVID-19 severity correlates with airway

10 epithelium-immune cell interactions identified by single-cell analysis. Nat Biotechnol 38, 970-

11979.

12 Corman, V.M., Landt, O., Kaiser, M., Molenkamp, R., Meijer, A., Chu, D.K.W., Bleicker, T.,

13 Brunink, S., Schneider, J., Schmidt, M.L., et al. (2020). Detection of 2019 novel coronavirus

14 (2019-nCoV) by real-time RT-PCR. Euro Surveill 25.

15 de Wit, E., Rasmussen, A.L., Falzarano, D., Bushmaker, T., Feldmann, F., Brining, D.L.,

16 Fischer, E.R., Martellaro, C., Okumura, A., Chang, J., et al. (2013). Middle East respiratory

17 syndrome coronavirus (MERS-CoV) causes transient lower respiratory tract infection in rhesus

18 macaques. Proc Natl Acad Sci U S A 110, 16598-16603.

19 Edgar, R., Domrachev, M., and Lash, A.E. (2002). Gene Expression Omnibus: NCBI gene

20 expression and hybridization array data repository. Nucleic Acids Res 30, 207-210. 
1 Feldmann, F., Shupert, W.L., Haddock, E., Twardoski, B., and Feldmann, H. (2019). Gamma

2 Irradiation as an Effective Method for Inactivation of Emerging Viral Pathogens. Am J Trop Med

$3 \quad$ Hyg 100, 1275-1277.

4 Ge, H., Wang, X., Yuan, X., Xiao, G., Wang, C., Deng, T., Yuan, Q., and Xiao, X. (2020). The

5 epidemiology and clinical information about COVID-19. Eur J Clin Microbiol Infect Dis 39, 1011 -

61019.

7 Gerner, M.Y., Kastenmuller, W., Ifrim, I., Kabat, J., and Germain, R.N. (2012). Histo-cytometry:

8 a method for highly multiplex quantitative tissue imaging analysis applied to dendritic cell subset

9 microanatomy in lymph nodes. Immunity 37, 364-376.

10 Han, X., Wang, R., Zhou, Y., Fei, L., Sun, H., Lai, S., Saadatpour, A., Zhou, Z., Chen, H., Ye, F., 11 et al. (2018). Mapping the Mouse Cell Atlas by Microwell-Seq. Cell 173, 1307.

12 Harcourt, J., Tamin, A., Lu, X., Kamili, S., Sakthivel, S.K., Murray, J., Queen, K., Tao, Y.,

13 Paden, C.R., Zhang, J., et al. (2020). Severe Acute Respiratory Syndrome Coronavirus 2 from

14 Patient with 2019 Novel Coronavirus Disease, United States. Emerg Infect Dis 26.

15 Hartman, A.L., Nambulli, S., McMillen, C.M., White, A.G., Tilston-Lunel, N.L., Albe, J.R., Cottle,

16 E., Dunn, M., Frye, L.J., Gilliland, T.H., et al. (2020). SARS-CoV-2 infection of African green

17 monkeys results in mild respiratory disease discernible by PET/CT imaging and prolonged

18 shedding of infectious virus from both respiratory and gastrointestinal tracts. bioRxiv. 
1 Huang, C.G., Lee, K.M., Hsiao, M.J., Yang, S.L., Huang, P.N., Gong, Y.N., Hsieh, T.H., Huang,

2 P.W., Lin, Y.J., Liu, Y.C., et al. (2020). Culture-Based Virus Isolation To Evaluate Potential

3 Infectivity of Clinical Specimens Tested for COVID-19. J Clin Microbiol 58.

4 Kim, D., Lee, J.Y., Yang, J.S., Kim, J.W., Kim, V.N., and Chang, H. (2020). The Architecture of 5 SARS-CoV-2 Transcriptome. Cell 181, 914-921 e910.

6 Liao, M., Liu, Y., Yuan, J., Wen, Y., Xu, G., Zhao, J., Cheng, L., Li, J., Wang, X., Wang, F., et al. 7 (2020). Single-cell landscape of bronchoalveolar immune cells in patients with COVID-19. Nat $8 \quad$ Med 26, 842-844.

9 Lucas, C., Wong, P., Klein, J., Castro, T.B.R., Silva, J., Sundaram, M., Ellingson, M.K., Mao, T., 10 Oh, J.E., Israelow, B., et al. (2020). Longitudinal analyses reveal immunological misfiring in 11 severe COVID-19. Nature.

12 Madissoon, E., Wilbrey-Clark, A., Miragaia, R.J., Saeb-Parsy, K., Mahbubani, K.T.,

13 Georgakopoulos, N., Harding, P., Polanski, K., Huang, N., Nowicki-Osuch, K., et al. (2019).

14 scRNA-seq assessment of the human lung, spleen, and esophagus tissue stability after cold 15 preservation. Genome Biol 21, 1. McAuliffe, J., Vogel, L., Roberts, A., Fahle, G., Fischer, S., Shieh, W.J., Butler, E., Zaki, S., St

17 Claire, M., Murphy, B., et al. (2004). Replication of SARS coronavirus administered into the 18 respiratory tract of African Green, rhesus and cynomolgus monkeys. Virology 330, 8-15.

19 Menter, T., Haslbauer, J.D., Nienhold, R., Savic, S., Hopfer, H., Deigendesch, N., Frank, S., 20 Turek, D., Willi, N., Pargger, H., et al. (2020). Postmortem examination of COVID-19 patients 
1 reveals diffuse alveolar damage with severe capillary congestion and variegated findings in

2 lungs and other organs suggesting vascular dysfunction. Histopathology.

3 Munster, V.J., Feldmann, F., Williamson, B.N., van Doremalen, N., Perez-Perez, L., Schulz, J.,

4 Meade-White, K., Okumura, A., Callison, J., Brumbaugh, B., et al. (2020). Respiratory disease

5 in rhesus macaques inoculated with SARS-CoV-2. Nature.

6 Qi, F., Qian, S., Zhang, S., and Zhang, Z. (2020). Single cell RNA sequencing of 13 human

7 tissues identify cell types and receptors of human coronaviruses. Biochem Biophys Res

8 Commun 526, 135-140.

9 Robinson, J.T., Thorvaldsdottir, H., Winckler, W., Guttman, M., Lander, E.S., Getz, G., and 10 Mesirov, J.P. (2011). Integrative genomics viewer. Nat Biotechnol 29, 24-26.

11 Sergushichev, A. (2016). An algorithm for fast preranked gene set enrichment analysis using 12 cumulative statistic calculation. bioRxiv.

13 Stuart, T., Butler, A., Hoffman, P., Hafemeister, C., Papalexi, E., Mauck, W.M., 3rd, Hao, Y., 14 Stoeckius, M., Smibert, P., and Satija, R. (2019). Comprehensive Integration of Single-Cell 15 Data. Cell 177, 1888-1902 e1821.

16 Subramanian, A., Tamayo, P., Mootha, V.K., Mukherjee, S., Ebert, B.L., Gillette, M.A., 17 Paulovich, A., Pomeroy, S.L., Golub, T.R., Lander, E.S., et al. (2005). Gene set enrichment 18 analysis: a knowledge-based approach for interpreting genome-wide expression profiles. Proc 19 Natl Acad Sci U S A 102, 15545-15550. 
1 Sungnak, W., Huang, N., Becavin, C., Berg, M., Queen, R., Litvinukova, M., Talavera-Lopez, C.,

2 Maatz, H., Reichart, D., Sampaziotis, F., et al. (2020). SARS-CoV-2 entry factors are highly

3 expressed in nasal epithelial cells together with innate immune genes. Nat Med 26, 681-687.

4 Tay, M.Z., Poh, C.M., Renia, L., MacAry, P.A., and Ng, L.F.P. (2020). The trinity of COVID-19:

5 immunity, inflammation and intervention. Nat Rev Immunol 20, 363-374.

6 Te Velthuis, A.J.W., Long, J.C., Bauer, D.L.V., Fan, R.L.Y., Yen, H.L., Sharps, J., Siegers, J.Y.,

7 Killip, M.J., French, H., Oliva-Martin, M.J., et al. (2018). Mini viral RNAs act as innate immune

8 agonists during influenza virus infection. Nat Microbiol 3, 1234-1242.

9 Tian, S., Xiong, Y., Liu, H., Niu, L., Guo, J., Liao, M., and Xiao, S.Y. (2020). Pathological study

10 of the 2019 novel coronavirus disease (COVID-19) through postmortem core biopsies. Mod

11 Pathol 33, 1007-1014.

12 Wakida, H., Kawata, K., Yamaji, Y., Hattori, E., Tsuchiya, T., Wada, Y., Ozaki, H., and Akimitsu,

13 N. (2020). Stability of RNA sequences derived from the coronavirus genome in human cells.

14 Biochem Biophys Res Commun 527, 993-999.

15 Wen, W., Su, W., Tang, H., Le, W., Zhang, X., Zheng, Y., Liu, X., Xie, L., Li, J., Ye, J., et al.

16 (2020). Immune cell profiling of COVID-19 patients in the recovery stage by single-cell

17 sequencing. Cell Discov 6, 31.

18 Wickham, H. (2016). ggplot2: Elegant Graphics for Data Analysis (Springer-Verlag New York). 
1 Wilk, A.J., Rustagi, A., Zhao, N.Q., Roque, J., Martinez-Colon, G.J., McKechnie, J.L., Ivison,

2 G.T., Ranganath, T., Vergara, R., Hollis, T., et al. (2020). A single-cell atlas of the peripheral

3 immune response in patients with severe COVID-19. Nat Med 26, 1070-1076.

4 Wolfel, R., Corman, V.M., Guggemos, W., Seilmaier, M., Zange, S., Muller, M.A., Niemeyer, D.,

5 Jones, T.C., Vollmar, P., Rothe, C., et al. (2020). Virological assessment of hospitalized patients

6 with COVID-2019. Nature 581, 465-469.

7 Woolsey, C., Borisevich, V., Prasad, A.N., Agans, K.N., Deer, D.J., Dobias, N.S., Heymann,

8 J.C., Foster, S.L., Levine, C.B., Medina, L., et al. (2020). Establishment of an African green

9 monkey model for COVID-19. bioRxiv. 